$16^{\text {th }}$ International Congress of Metrology, 13001 (2013)

DOI: $10.1051 /$ metrology/201313001

(C) Owned by the authors, published by EDP Sciences, 2013

\title{
Nanoscale Transport Measurements with Multiple Probe Scanning Tunneling Microscopy
}

\author{
M. Berthe, C. Durand, R. Leturcq, B. Grandidier \\ Institut d'Electronique, de Microélectronique et de Nanotechnologie, IEMN, (CNRS, UMR 8520), Département ISEN, 41 \\ bd Vauban, 59046 Lille Cedex, France
}

\begin{abstract}
Development of nanometer-sacle probing techniques have become imperative for current flow and resistivity measurements with nanometer scale spatial resolution in a variety of materials and devices. The use of weakly coupled scanning tunneling probes to detect transport phenomena appears to be an appropriate approach. We will describe a new setup, where the unique combination of four scanning tunneling microscope probe tips and a scanning electron microscope in ultra high vacuum allows 4-point contact measurements and function testing of nanodevices within complex structures and integrated circuits. Such a tool has considerable potential, as it does not need any extra electrodes on the specimen and enables arbitrary arrangements of probes, with the highest spatial resolution, on isolated semiconductor nanowires, for example.
\end{abstract}

\section{Introduction}

In a wide range of semiconductor applications the characterization of the local conductivity is crucial for the final device performance. To fulfill this goal, four-point probe measurements have been a technique of choice, due to the low requirements on sample preparation and its high accuracy. The measurements are mostly done on large samples - test-wafers - with dimensions much larger than the pitch of the four-point probes. With the increasing wafer size and processing costs, considerable savings may be realized if accurate characterization can be done on small dedicated areas on device wafers instead.

For a large conductor, the probes only represent a minor perturbation. But with the ongoing reduction in sizes of devices, standard probes can become very invasive and the dominant source of scattering at the nanoscale. Therefore, the use of probes operated with a scanning tunneling microscopy has considerable potential $[1,2,3,4,5]$, as it does not need any extra electrodes on the specimen and enables arbitrary arrangements of probe electrodes with the highest spatial resolution. Here, we will first give a brief description of an instrument that combines multiple probe scanning tunneling microscopy (STM) with scanning electron microscopy (SEM) in ultra high vacuum. Based on the atomic precision of motion control of STM and real-time and fast imaging capability of SEM, we will further show how this instrument can be used to characterize semiconductor nanowires prior to their integration into devices.

\section{Instrumentation}

In STM, measurable tunneling currents usually ranges from tens of femtoamperes to hundreds of nanoamperes, depending on the width of the tunneling barrier and the conductivity of the materials. To achieve the appropriate tip-sample separation, actuators, consisting of piezoeletric ceramics that expand or contract almost linearly with the voltage applied across the ceramics, are required to move the tip with a precision of the order of the picometer. In the case of a four-probe STM, four STM probes are mounted on four different cylindrical piezo actuators that can be translated independently by inertial motion driving systems. The whole plateform (see Figure 1) is surmounted with a SEM [6], that enables a large field of view for probe coarse positioning as well as fine positioning and rapid localisation of nanometersized structures. When the probe tip apex shadows nanometer-sized structures in the SEM navigation or if the structures are even smaller than accessible by SEM, the atomic scale precision of STM allows the final precise positioning of the probe tip. In order to begin experiments with a reproducibly clean sample, and to ensure that significant contamination by background gases does not occur during an experiment, the whole instrument works in ultra high vacuum (UHV). Finally, the instrument is equipped with fast-entry load locks and a preparation chamber. This extension allows for separate tip and sample preparation with a wide range of different processes such as sputtering, annealing, and thin film deposition.

\footnotetext{
$\bar{a}$ Corresponding author: author@email.org
} 


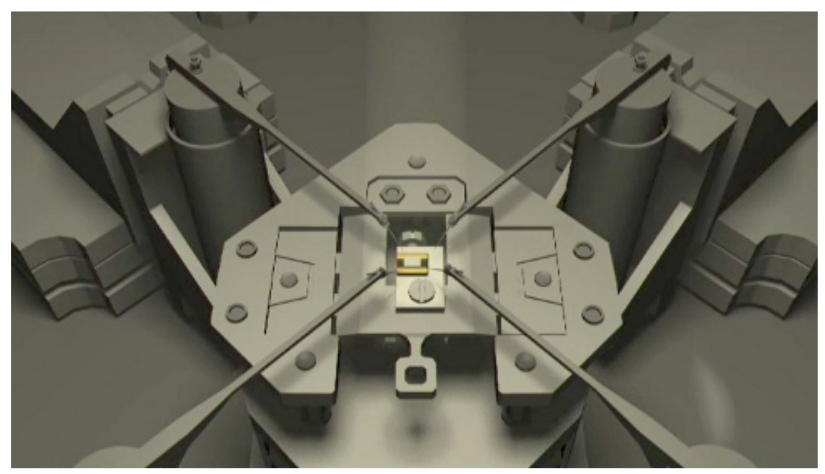

Figure 1. Schematic diagram of the measurement stage of a four-probe scanning tunneling microscope, where the four STM tips are positioned above the sample, at the end of four actuactors, that are themselves supported by four coarse positioning systems.

To fully take advantage of the equipment, the SEM and all four STM tips should be simultaneously and independently operated. Moreover, the STM tips can work in two different modes, either in the tunneling regime, where they are used to observe a sample and to perform tunneling spectroscopic measurements, or in the contact regime, where they are used in a source/measure configuration to perform DC electrical measurements. Due to the complexity that can occur when multiple probes are operated at the same time, a powerful control system that allows all types of experiments was built. As measurements of the conductivity of a nanoscale component require to bring the STM tips into contact, each feedback loop used for imaging the sample with the STM can be disabled by switching units. These modules provide an alternative output to the STM tips that can be connected to other preamplifiers, voltage or current sources, electrical measurement devices, function generators, etc.

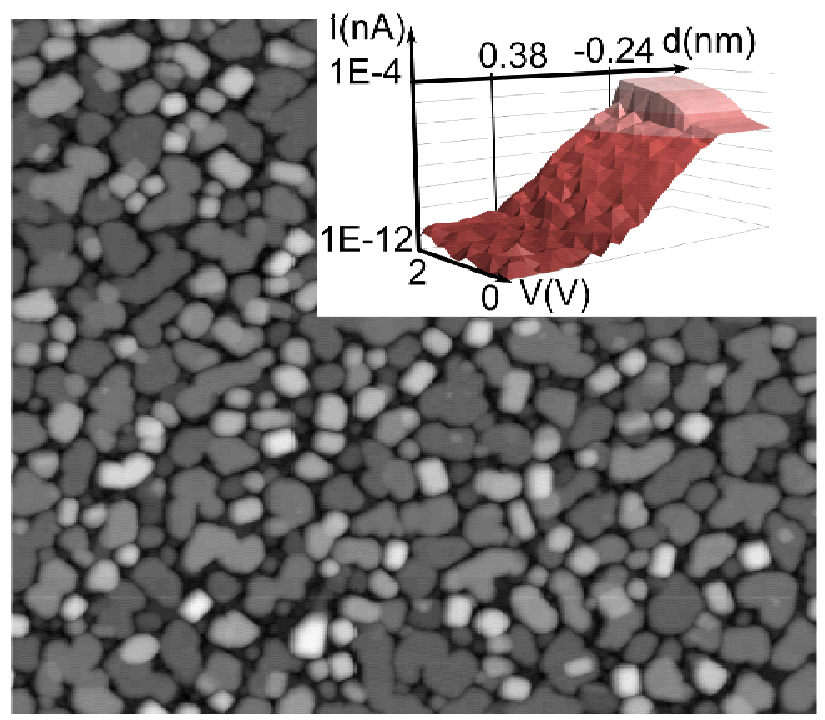

Figure 2. STM image of Al nanodots grown on silicon (image width: $120 \mathrm{~nm})$. Inset: $\mathrm{I}(\mathrm{V}, \mathrm{d})$ characteristics acquired on a dot, by varying the sample voltage $\mathrm{V}$ and the tip sample distance $\mathrm{d}$ spectroscopic measure-ments acquired on a dot, by varying the sample voltage and the tip sample distance. The low/high current regions correspond to tunneling /contact regimes.
Figure 2 shows a STM image of Al dots grown on a Si(111) surface. One of the STM tip was immobilized over a single dot and the signal switched to a low current source meter unit to record the different regimes of conductance as a function of the distance between tip and sample. The graph (vertical logarithmic scale) reports three regimes depending on the tip-sample distance. At large distances, when the tip is retracted from the sample, no current flows for any value of the voltage $\mathrm{V}$. At smaller distances $(0.38 \mathrm{~nm}>\mathrm{d}>-0.24 \mathrm{~nm})$, the $\mathrm{I}-\mathrm{V}$ characteristic corresponds to a straight line on the logarithmic scale, implying that the current varies exponentially as a function of the tip-sample distance. Then, for short tip-sample distances $(\mathrm{d}<-0.24 \mathrm{~nm})$, the current reaches saturation, due when the tip gets in contact with the dot. In this regime, if the tip-sample distance is kept constant, a rectifier-shaped I(V) curve is measured when the bias varies. This behaviour is caused by the formation of a Schottky barrier between the Si surface and the Al dot. Such a result demonstrates the potential of each probe to inject or measure a current with both spatial and electrical precision.

\section{Resistivity of InAs nanowires}

Narrow band gap materials span over the sub-eV spectrum and have opened the way to a multitude of applications that range from infrared optoelectronics $[7,8]$ to low-power logic applications $[9,10]$ and quantum information [11]. While two-dimensional heterostructures made up of these materials generally suffer from extreme lattice mismatch, the successful growth of nanowires with improved crystal structure offers new perspectives in integrating these materials into fast and low-power transistors [12]. Since the ratio between surface and volume in one-dimensional systems becomes important with respect to the bulk crystal, their transport properties might be governed by the surface structure and chemistry of their sidewalls $[13,14]$. Therefore, extensive studies of their conductivity with precise control over the nanowire surface structure and its environment are required to determine the contribution of the surface to the electronic transport.

[0001]-oriented wurtzite InAs NWs were grown from gold droplets by gas source molecular beam epitaxy, technique that is known to significantly limit the bulk incorporation of impurities in III-V NWs. Starting with an InP stem growth at $410^{\circ} \mathrm{C}$ on $\operatorname{InP}(111) \mathrm{B}$ substrates, InAs nanowires were subsequently grown by direct switch from $\mathrm{P}_{2}$ to $\mathrm{As}_{2}$ molecular fluxes. The NW mean diameter was $70 \pm 9 \mathrm{~nm}$. Three types of geometry were electrically investigated: interconnected NWs with conventional planar technology, free-standing NWs and flat-lying NWs. For the first ones, NWs were deposited onto a degenerately-doped $\mathrm{Si}$ wafer with a $225 \mathrm{~nm}$ of thermally-grown $\mathrm{SiO}_{2}$ layer and selected for contact processing with a scanning electron microscope at low magnification and with a short integration time. After spin-coating the sample with an electron-beam sensitive polymer, four contacts are defined along the nanowire using electron beam exposure. The oxide on the contact 
area was etched in a $\left(\mathrm{NH}_{4}\right)_{2} \mathrm{~S}_{\mathrm{x}}$ solution just before evaporation of $\mathrm{Ti} / \mathrm{Au}$ electrodes (10/150 $\mathrm{nm}$ thick). This step was followed by a thorough lift-off to get NWs with clean oxidized sidewalls. In this case, a CCD camera mounted on the UHV system was used to position the STM tips on metallic pads, thus avoiding the exposure of the NWs to the e-beam (see Figure 3(a) and (b)). As to the second type of geometry, the as-grown NWs were manipulated into direct contact with the substrate used to interconnect NWs. Based on this action, the NWs were cleaved and falled down flat on the $\mathrm{SiO}_{2}$ surface [15]. There, they could be contacted with four STM tips, as shown in Figure 3(c).
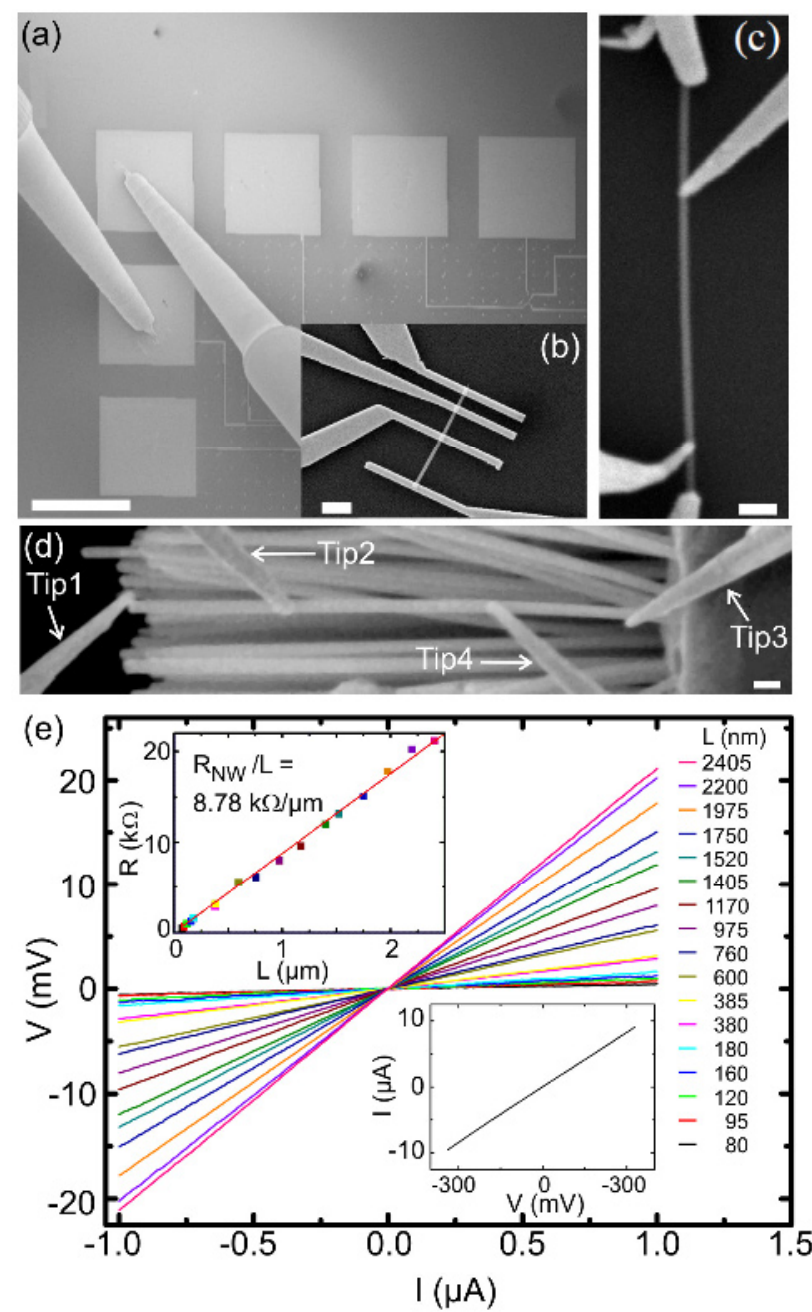

Figure 3. (a) Large scale SEM image of the sample geometry used to characterize the electrical transport in interconnected InAs nanowires. Two STM tips are plugged to the source and drain electrodes. Scale bar: $300 \mu \mathrm{m}$. (b) SEM enlarged view of the InAs NW in electrical contact with four electrodes Scale bar: $1 \mu \mathrm{m}$. (c) SEM image of a single InAs $\mathrm{NW}$ on a $\mathrm{SiO}_{2}$ surface in contact with four STM tips. Scale bar: $300 \mathrm{~nm}$ (d) SEM image of free-standing InAs NWs with clean and wellordered sidewalls that were grown on a $\operatorname{InP}(111) \mathrm{B}$ substrat (right part of the image). The NW in the centre is contacted by four $\mathrm{W}$ tips. (e) $V-I$ curves measured with the four probe method for different spacings $L$ between tips 2 and 4 . The length $L$ is given in the right of the graph. Upper inset: Resistance measured from the $V-I$ measurements as a function of $L$. Lower inset: $I-V$ curve measured between tip 2 and the substrate. Scale bar : $150 \mathrm{~nm}$.
Finally, in the third configuration, the as-grown NWs were kept on the InP substrate. It allows to keep the crystalline surface of the NWs intact, when the samples are capped with a thin layer of As at the end of the growth. Such a capping layer protects the NW surface from oxidation when the samples are transferred to air. It can be desorbed in UHV at $350^{\circ} \mathrm{C}$, monitoring the As desorption with mass spectrometry [16]. Figure 3(d) shows a typical array of free-standing InAs NWs, where the NWs have a mean diameter of $70 \mathrm{~nm}$ and an average length of $2.5 \mu \mathrm{m}$. Thanks to the precision of the STM scanners, four tips have been brought into electrical contacts with the NW visible in the centre of the SEM image. In most cases, the I(V) curve between a single tip and the substrate, held at ground, has an ohmic behaviour, as shown in the lower inset of Figure 3(e), where the resistance is $36 \mathrm{k} \Omega$. Tips 1 and 3 were respectively connected to the gold catalyst and the base of the wire and used as source and ground electrodes for driving the current through the wire, while the substrate was kept floating. Tips 2 and 4 were the potential probes.

When a current is passed through the wire, the potential drop measured between the inner probes is found to be linearly proportional to the current intensity (see Figure 3(e)). The gradient of the curves yields the four-probe resistance of the wires for a given separation between the inner probes. By moving the inner tips towards the center of the wire, a set of $V(I)$ characteristics was acquired for different tip separations $L$. From these $V(I)$ curves, we obtain the resistance $R_{\mathrm{NW}}$ that is found to linearly increase with $L$, as shown in the upper inset of Figure 3(e). The slope yields a resistance per unit length $R_{\mathrm{NW}} / L$ of $8.78 \mathrm{k} \Omega / \mu \mathrm{m}$.

\section{Conclusion}

We have demonstrated that a multiprobe STM allows us to measure the resistivity of free-standing semiconductor NWs. Comparison of the resistivity between those free-standing InAs NWs and nonirradiated InAs NWs has more recently showed a significant and reproducible decrease of the resistivity upon irradiation [17]. This effect is caused by the formation of a deep accumulation layer at the surface of the NWs due to the generation of defects on the InAs NW sidewalls. As surface charged defects induced by electron irradiation should occur in all types of semiconductor nanomaterials, causing more or less noticeable changes in their transport properties as a function of the defect concentrations, this study points to additional cautions during the controlled positioning of nanostructures in devices and the subsequent inspection of their integrity when high resolution analytical and imaging techniques, such as SEM, are used.

\section{Acknowledgment}

We thank Ph. Caroff for the growth of the nanowires and his strong support. 


\section{References}

1. S. Hasegawa, I. Shiraki, F. Tanabe, R. Hobara, T. Kanagawa, T. Tanikawa, I. Matsuda, C. L. Petersen, T. M. Hansen, P. Boggild, F. Grey, Surf. Rev. Lett. 6, 963 (2003).

2. T. Kanagawa, R. Hobara, I. Matsuda, T. Tanikawa, A. Natori, S. Hasegawa, Phys. Rev. Lett. 91, 36805 (2003).

3 P. W. Sutter, J.-I. Flege, E.A. Sutter, Nature Materials 7, 406 (2008).

4. T.-H. Kim, X.-G. Zhang, D. M. Nicholson, B. M. Evans, N. S. Kulkarni, B. Radhakrishnan, E. A. Kenik, A.-P. Li, Nano Lett. 10, 3096 (2010).

5. S.-H. Ji, J. B. Hannon, R. M. Tromp, V. Perebeinos, J. Tersoff, F. M. Ross, Nature Materials 11, 114 (2011).

6. Nanoprobe, Omicron Nanotechnology

7. O. B. Shchekin, and D. G. Deppe, Appl. Phys. Lett. 80, 3277 (2002).

8. H. Lim, S. Tsao, W. Zhang, and M. Razeghi, Appl. Phys. Lett. 90, 131112 (2007).

9. J.B. Boos, W. Kruppa, B. R. Bennett, D. Park, S. W. Kirchoefer, R. Bass, and H. B. Dietrich, IEEE Trans. Electron Dev. 45, 1869 (1998).

10. B. R. Bennett, R. Magno, J. B. Boos, W. Kruppa, and M. G. Ancona, Solid-State Electron. 49, 1875 (2005). 11. A. Kiraz, P. Michler, C. Becher, B. Gayral, A. Imamoglu, L.Zhang, E. Hu, W. V. Schoenfeld, and P. M. Petroff, Appl. Phys. Lett. 78, 3932 (2001).

12. P. Caroff, M. E. Messing, B. M. Borg, K. A. Dick, K. Deppert, and L.-E. Wernersson, Nanotechnol. 20, 495606 (2009).

13. A. C. Ford, J. C. Ho, Y.-L. Chueh, Y.-C. Tseng, Z. Fan, J. Guo, J. Bokor and A. Javey, Nano Lett. 9, 360 (2009).

14. A. M. Katzenmeyer, F. Léonard, A. A. Talin, M. E. Toimil-Molares, and J. G. Cederberg, IEEE Trans. Nanotechnol. 10, 92 (2011).

15. T. Xu, J. P. Nys, A. Addad, O. I. Lebedev, A. Urbieta, B. Salhi, M. Berthe, B. Grandidier, D. Stiévenard Phys. Rev. B 81, 115403 (2010).

16. T. Xu, K. A. Dick, S. Plissard, T. H. Nguyen, Y. Makoudi, M. Berthe, J.-P. Nys, X. Wallart, B. Grandidier, P. Caroff, Nanotechnol. 23, 095702 (2012).

17. C. Durand, M. Berthe, Y. Makoudi, J.-P. Nys, R. Leturcq, P. Caroff, B. Grandidier, Nanotechnol. 24, 275706 (2013). 